Tropical Journal of Pharmaceutical Research June 2012; 11 (3): 361-369

(C) Pharmacotherapy Group, Faculty of Pharmacy, University of Benin

Benin City, 300001 Nigeria.

All rights reserved.

Available online at http://www.tjpr.org

Research Article

http://dx.doi.org/10.4314/tjpr.v11i3.4

\title{
Formulation of Fast-Release Gastroretentive Solid Dispersion of Glibenclamide with Gelucire 50/13
}

\author{
Prashant Upadhyay ${ }^{1^{*}}$ and Jayanta Kumar Pandit ${ }^{2}$ \\ ${ }^{1}$ Department of Pharmaceutics, College of Pharmacy, I.F.T.M, Delhi Road, Moradabad-244001 and Gautam Buddh \\ Technical University, Lucknow, ${ }^{2}$ Department of Pharmaceutics, Institute of Technology, Banaras Hindu University, \\ Varanasi-221005, Uttar Pradesh, India
}

\begin{abstract}
Purpose: Fast-release gastroretentive solid dispersions of glibenclamide using gelucire were prepared to achieve improved bioavailability.

Methods: Hot melt granulation technique was adopted to prepare solid dispersions (SDs) of glibenclamide in gelucire 50/13 and were compared with pure glibenclamide and physical mixtures of drug and gelucire using hot stage polarized microscopy, powder x-ray diffraction (PXRD), Fouriertransform infrared spectroscopy FTIR, bouyancy as well as by in vitro release and in vivo studies. Further aging studies were carried out for the samples.

Results: PXRD showed that glibenclamide was present in $S D$ in an amorphous form while FTIR spectroscopy revealed the presence of hydrogen bonding in the SDs. In vitro buoyancy was found for $11 \mathrm{~h}$ and there was improvement in solubility and dissolution rate for all test formulations. Formulations were found to follow Zero order kinetic. . During aging study, no decrease of in vitro drug dissolution was observed over 3-month period. Crystallinity in the SDs was observed following aging. A more pronounced lowering of blood glucose level in Wistar rats compared with the pure drug, suggests that the test formulations are superior.

Conclusion: This study demonstrates the high potential of hot melt technique for obtaining stable fastrelease gastroretentive solid dispersions of poorly water soluble drug using polyglycolized glycerides as carriers
\end{abstract}

Keywords: Glibenclamide, Gelucire, Solid dispersion, Gastro-retentive multi-particulates, Hot melt technique. 


\section{INTRODUCTION}

The gastro-residence time of orally administered dosage form is generally short due to rapid gastric emptying. Rapid gastrointestinal transit could result in incomplete drug release from orally administered dosage form above the absorption zone, leading to diminished efficacy [1]. In order to increase the bioavailability of such drugs, the residence time of the orally administered dosage form in the upper gastrointestinal tract needs to be prolonged. The approaches to prolong gastroresidence time of pharmaceutical dosage forms include bioadhesive, mucoadhesive and density control delivery system [2-4].

Recently, much attention has been focused on the use of fats and fatty acid as carriers in drug delivery systems [5-7]. Mahadik et al [8] demonstrated the use of amphiphilic lipid glyceryl monooleate for the design of floating matrix system. Gelucire is in the family of vehicles derived from mixtures of mono-, diand tri-glycerides with PEG esters of fatty acids. These are available with a range of properties depending on their hydrophiliclipophilic balance (HLB) value and melting point range $\left(33-65^{\circ} \mathrm{C}\right)$. These are used in the preparation of fast release and sustained release formulations. Gelucire containing only PEG esters are generally used in the preparation of fast release formulation. Owing to their extreme hydrophilicity and low density, Gelucire 50/13 may be considered an appropriate carrier for designing a fast release floating drug delivery system [9]. Glibenclamide is a poorly soluble drug with possible content uniformity problems and dissolution rate-limited bioavailability, is given in a therapeutic dose of $5-15 \mathrm{mg}$ daily, has a half-life of around $10 \mathrm{~h}$ and exhibits low bioavailability. However, the bioavailability of the drug has been found to reduce further with conventional dosage forms probably due to the fact that passage of the single unit dosage form of the drug is faster than its release and most of the drug is released in the colon [10]. Therefore, it is desirable to improve on earlier formulations by developing fast release gastroretentive solid dispersion system. Solubility enhancement of glibenclamide is an important aspect of formulation development. Although there is a plethora of reports of solubility improvement using different techniques, a comparative study of different solubilization approaches are few [11]. Solubilization of poorly aqueous soluble drug forms an important activity in formulation process [12]. Therefore, the objective of this study was to design and develop, with the aid of solubilizers, glibenclamide formulation with enhanced solubility using a solid dispersion approach.

\section{EXPERIMENTAL}

\section{Materials}

Glibenclamide was received as a gift from Ranbaxy, Gurgaon, India. Gelucire 50/13 (semi-synthetic polyglycolized glycerides) was provided by Gattefosse, St.Priest, Cedex, France. All other materials and reagents used were of analytical grade.

\section{Preparation of solid dispersions (SDs) and physical mixtures (PMs)}

Solubility enhancement of glibenclamide (GLB) was achieved by dispersing the drug in molten Gelucire 50/13 at various ratios (1:1, 1:2, 1:4 and 1:10)[9]. It was poured on aluminium foil, allowed to solidify in a covered Petri dish and then kept in a refrigerator for 3 h. The solid lump was passed through a fine mesh $(150 \mu \mathrm{m})$ to obtain a fine powder formulation that was then placed in a calcium chloride desiccator for $48 \mathrm{~h}$. The quantities of GLB, SDs and hydrophilic additives, namely, polyethylene glycol (PEG) 200, 400, 4000 and 6000 used are as shown in Table 1. Physical mixtures (PMs) of drug and Gelucire were prepared by triturating them for $15 \mathrm{~min}$ followed by sieving through a $150 \mu \mathrm{m}$ mesh. 
Table 1: Composition (mg) of glibenclamide formulations containing Gelucire 50/13 and PEGs

\begin{tabular}{lccccccccc}
\hline Ingredient & GI & G II & G III & G IV & G V & G VI & G VII & G VIII & G IX \\
\hline GLB & 5 & 5 & 5 & 5 & 5 & 5 & 5 & 5 & 5 \\
PEG 200 & - & 5 & - & - & - & 10 & - & - & - \\
PEG 400 & - & - & 5 & - & - & - & 10 & - & - \\
PEG 4000 & - & - & - & 5 & - & - & - & 10 & - \\
PEG 6000 & - & - & - & - & 5 & - & - & - & 10 \\
Gelucire 50/13 & 50 & 50 & 50 & 50 & 50 & 50 & 50 & 50 & 50 \\
$\quad$ Total & 55 & 60 & 60 & 60 & 60 & 65 & 65 & 65 & 65 \\
\hline
\end{tabular}

\section{Determination of drug content of SDs}

SDs equivalent to $5 \mathrm{mg}$ of GLB were weighed accurately and dissolved in a suitable quantity of methanol. After filtration through a $0.45 \mu \mathrm{m}$ filter (Ashless-Whatman, England), drug content was determined at $229 \mathrm{~nm}$ by spectrophotometry (Shimadzu UV-1800, Japan) with suitable dilution. Absorbance determination was performed in triplicate.

\section{Evaluation of saturation solubility}

The saturation solubility of GLB, PMs and SDs was evaluated by adding known excess amount of GLB formulation or pure GLB to 10 $\mathrm{ml}$ of $0.1 \mathrm{M} \mathrm{HCl}(\mathrm{pH} \mathrm{1.2})$, stirred at $20 \mathrm{rpm}$ in a water bath $\left(25 \pm 0.3^{\circ} \mathrm{C}\right)$ for $48 \mathrm{~h}$, filtered, diluted suitably with $0.1 \mathrm{M} \mathrm{HCl}(\mathrm{pH} 1.2)$ and analyzed at $229 \mathrm{~nm}$.

\section{Hot stage polarized microscopy (HSPM)}

About $2 \mathrm{mg}$ of sample was placed on a glass slide and covered with cover slip. The slide was examined under optical microscope fitted with a hot stage, heated at a rate of $2^{\circ} \mathrm{C} / \mathrm{min}$ from room temperature to $50^{\circ} \mathrm{C}$ where it was held for $30 \mathrm{~min}$, and then cooled to $40^{\circ} \mathrm{C}$. It was held at this temperature for $30 \mathrm{~min}$. Polarized light microscopy was applied for the detection of crystallinity [13].

\section{Powder x-ray diffraction (PXRD)}

X-ray diffraction studies on GLB, Gelucire $50 / 13$, PMs and SDs were determined using a D8 Advance, Bruker AXS instrument with a nickel-filtered radiation. The samples were irradiated with monochromatized CuK (a) radiation $(1.542 \AA)$ and analyzed between $2^{\circ}$ and $50^{\circ} 2 \theta$ using a step scan mode (step width $=0.020^{\circ}(2 \theta)$, counting time $=0.5$ $\mathrm{s} / \mathrm{step}$ ). Diffraction peak (d) intensities and 2 $h$ values of the SDs patterns were compared to those of the pure materials in order to evaluate the physical form of GLB in the samples.

\section{Fourier-transform infrared spectroscopy (FTIR)}

FTIR spectra of the individual materials as well as SDs were obtained, after appropriate background subtraction, using FTIR-8400 spectrometer (Shimadzu, Japan). About 2-3 $\mathrm{mg}$ of the sample was triturated with dry potassium bromide, compressed into disc and scanned from $4000-400 \mathrm{~cm}^{-1}$.

\section{Evaluation of in-vitro buoyancy}

In vitro bouyancy assessment was performed using a USP dissolution apparatus type II by placing the SD in $900 \mathrm{ml}$ of $0.1 \mathrm{M} \mathrm{HCl}(\mathrm{pH}$ 1.2) at $37.0 \pm 0.5^{\circ} \mathrm{C}$ and then agitated with a paddle at $75 \mathrm{rpm}$ for $12 \mathrm{~h}$. After agitation, the lipid particles that floated on the surface of the medium as well as those that settled at the bottom of the flask were removed separately. The proportion of floating particles was evaluated [14].

\section{In vitro drug release studies}

USP type II (paddle) method was used with the aid of Electrolab dissolution tester (TDT06 N, India). The dissolution medium 
used was $900 \mathrm{~mL}$ of $0.1 \mathrm{M} \mathrm{HCl}(\mathrm{pH} 1.2)$ at 37 $\pm 0.5^{\circ} \mathrm{C}$ and stirred at $75 \mathrm{rpm}$. An aliquot $(5$ $\mathrm{ml}$ ) was withdrawn at predetermined time intervals for $4 \mathrm{~h}$ and replaced with same volume of fresh medium. The withdrawn sample was suitably diluted and analyzed using a spectrophotometer at $229 \mathrm{~nm}$; the test was also carried out on a commercial brand of fast-release glibenclamide tablet (Betanase ${ }^{\circledR}$, Cadila Healthcare Ltd, India).

\section{Analysis of release kinetic and drug release mechanism}

The release data obtained were treated according to zero-order, first-order, Higuchi [15] and Korsmeyer-Peppas's models [16] with the aid of PCP-Disso software (V3, Poona College of Pharmacy, Pune, India), in order to analyze the kinetics of drug release from the formulations. Higuchi (Eq 1) and Korsmeyer-Peppas (Eq 2) models were also applied to determine drug release mechanism.

$$
\mathrm{Q}=\mathrm{Kt}^{1 / 2}
$$

where $Q$ is the amount of drug released in time, $\mathrm{t}$, and $\mathrm{K}$ is the release constant.

$$
\mathrm{M}_{\mathrm{t}} / \mathrm{M}_{\infty}=\mathrm{Kt}^{\mathrm{n}}
$$

where $M_{t} / M_{\infty}$ is the fraction of drug released in time, $\mathrm{t}, \mathrm{K}$ is the structural and geometric constant, and $\mathrm{n}$ is the release exponent [17].

\section{Effect of ageing}

The SDs were stored at $30^{\circ} \mathrm{C} / 65 \% \mathrm{RH}$ for 3 months and the effect of ageing on the SDs were studied by measuring their in vitro release as well as structural features using DSC and XRPD.

\section{Evaluation of blood glucose}

Blood glucose level (BGL) lowering studies of the SDs and pure GLBs were determined in streptozotocin (STZ)-induced diabetic Wistar rats of either sex weighing $150-200 \mathrm{~g}$. The animals were handled as per CPCSEA Guidelines of Good Laboratory Practice (GLP) [18]. They were housed in polypropylene cages with free access to standard laboratory diet and water. The research protocol of the animal experimentation (registration no. 837/ac/04/ CPCSEA, resolution no. 20/PhD/2008-2009, dated 8 March 2010) was approved by the 'Institutional Animal Ethical Committee' of College of Pharmacy, IFTM, Moradabad244001, Uttar Pradesh, India. The animals were divided into 4 groups. Group I (control) was given $2 \mathrm{ml}$ saline p.o. Diabetic control (Group II) was given STZ $35 \mathrm{mg} / \mathrm{kg}$ i.p. in citrate buffer $(\mathrm{pH}$ 4.5). Diabetic standard treatment Group III was given pure GLB $(0.25$ $\mathrm{mg} / \mathrm{kg}$ ) in aqueous solution p.o through an oral cannula. Diabetic test treatment (Group IV) was given GLB formulation (optimized batch) equivalent to $0.25 \mathrm{mg} / \mathrm{kg}$ of GLB p.o. in $2 \mathrm{ml}$ of $1 \%$ sodium carboxylmethyl cellulose via an oral cannula. Blood glucose level (BGL) was measured periodically using Ascensia Entrust Glucometer (Bayer HealthCare, USA).

\section{Statistical analysis}

Statistical analysis of the data was carried out by Student's t-test and one-way analysis of variance (ANOVA) at a significance level of $p$ $<0.05$ using SPSS 12.0 software (IBM).

\section{RESULTS}

\section{Saturation solubility}

The saturation solubility of GLB was 18.9 $\mu \mathrm{g} / \mathrm{ml}$ while the enhanced saturated solubility obtained using drug:Gelucire (1:1) in physical mixture and solid dispersion was 27.23 and $44.39 \mu \mathrm{g} / \mathrm{ml}$, respectively. Drug solubility increased in direct proportion to the proportion of Gelucire 50/13 in the preparations. Based on saturation solubility, ratio 1:10 showed enhanced solubility.

\section{Hot stage polarized microscopy (HSPM)}

HSPM of the SDs showed continuous melting from room temperature to $50^{\circ} \mathrm{C}$ and when it was cooled back to $40{ }^{\circ} \mathrm{C}$. Change in physical form was observed at different 

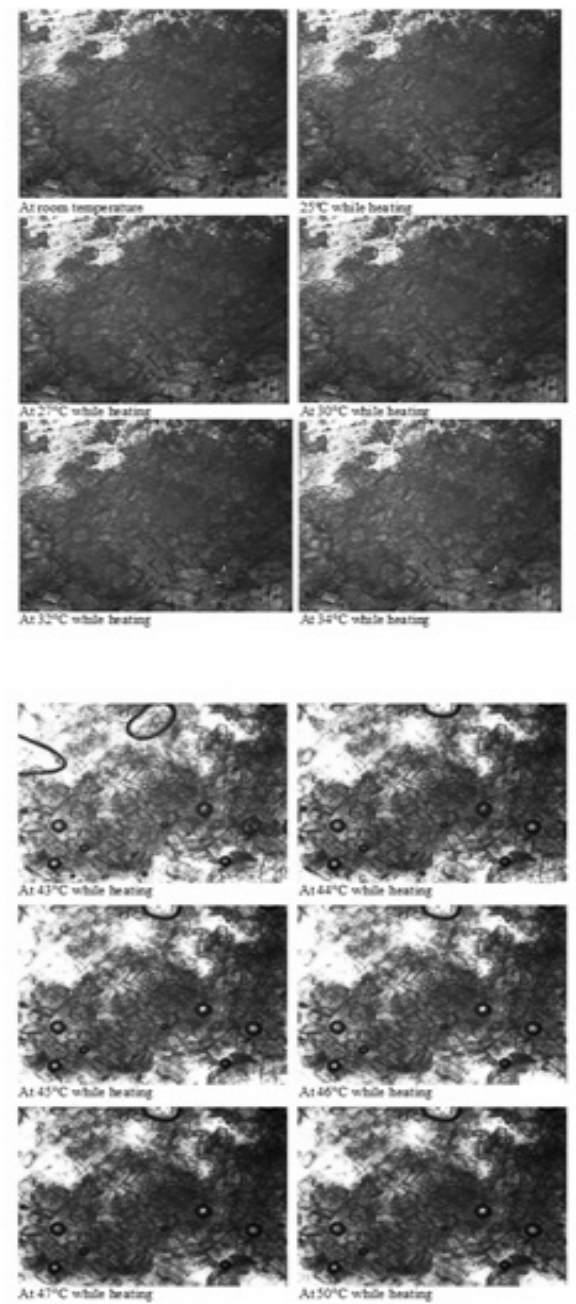
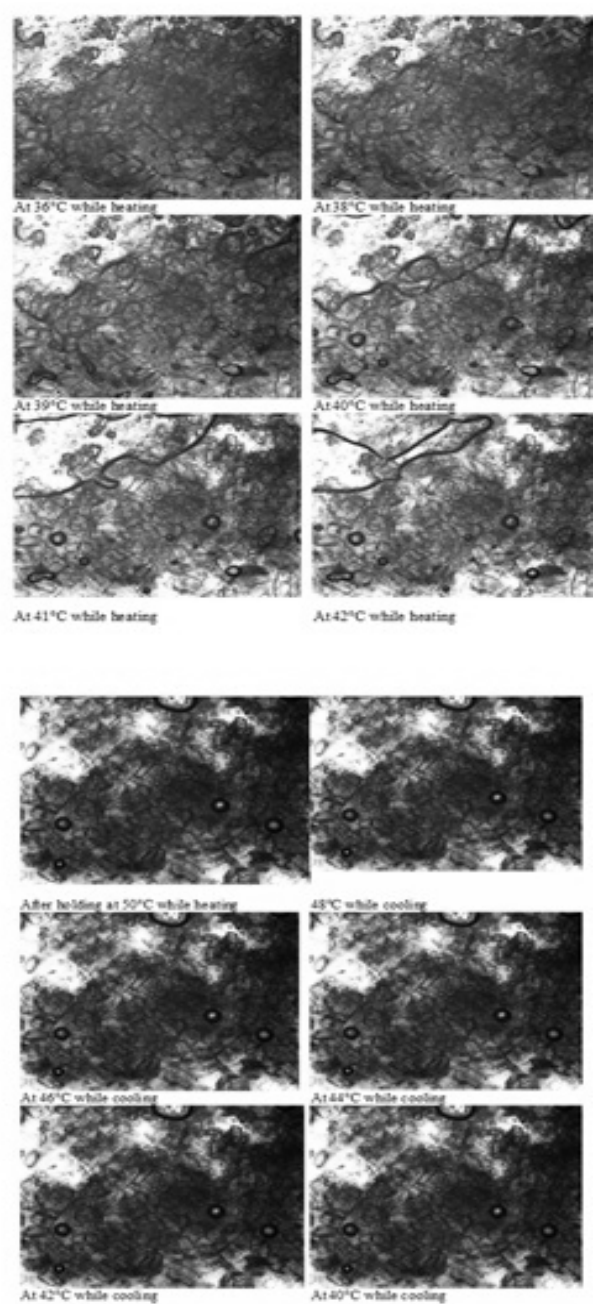

Fig 1: Typical photomicrographs of solid dispersions (SDs) showing physical changes as temperature is varied

temperatures as shown in the photomicrographs in Fig 1.. The photomicrographs indicate that large crystals of pure GLB were reduced to small particle size when they came in close contact with the hydrophilic carrier.

\section{X-ray diffraction}

The x-ray diffractograms of pure GLB (Fig 2a) had prominent diffraction peaks (d) equal to $8.035^{\circ}, 7.464^{\circ}, 4.649^{\circ}, 3.860^{\circ}, 2.933^{\circ}$ and $1.687^{\circ}$, respectively on $2 \theta$ scale, which indicates its crystalline nature. The diffractograms for the formulations and Gelucire 50/13 (Fig 2b) indicate that GLB peaks decreased, suggesting its conversion from a crystalline to an amorphous state; diffractograms for the formulations physical mixtures and solid dispersions (Fig 2 (c) and (d)) indicate that GLB peaks decreased, suggesting its conversion from crystalline to an amorphous state; Gelucire 50/13 showed two prominent diffraction peaks (d) of $4.61^{\circ}$ and $3.81^{\circ}$ with the highest intensity on $2 \theta$ scale. The principal peaks of Gelucire 50/13 
were present in both PMs and SDs with a lower intensity. The diffractogram of SDs showed absence of any trace of crystallinity, indicating the existence of amorphous GLB.
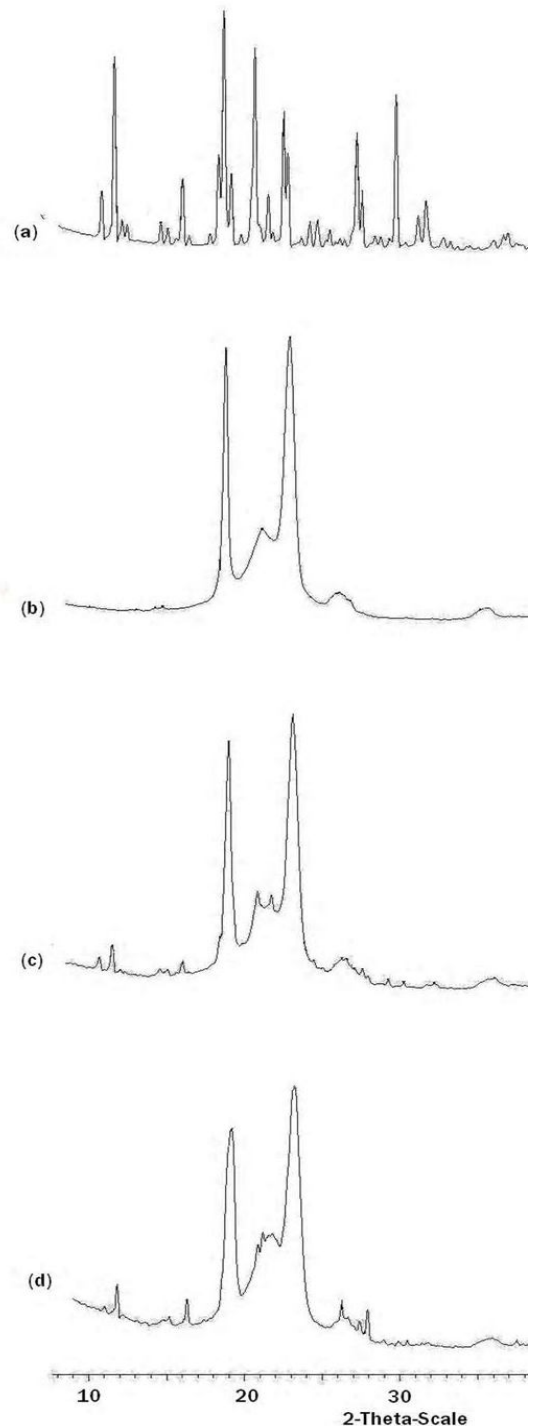

Fig 2: X-ray diffractogram of (a) pure GLB, (b) Gelucire 50/13, (c) physical mixtures (PMs) and (d) solid dispersions (SDs)

\section{Fourier transform infrared (FTIR) spectra}

FTIR spectra of pure GLB showed characteristic amide peaks at 3367.48 , $3315.41,2929.67,2854.45$ and $1716.53 \mathrm{~cm}^{-}$ 1; urea carbonyl stretching (urea $\mathrm{NH}$ stretching) vibration at 1618.2 and 1521.73 $\mathrm{cm}^{-1}$; and $\mathrm{SO}_{2}$ stretching vibration at 1340.43 and $1159.14 \mathrm{~cm}^{-1}$. On the other hand, the FTIR spectra of PMs showed slight intense amide peaks at 3315.41 and $1716.53 \mathrm{~cm}^{-1}$, while SDs showed almost complete disappearance of amide peaks at 3315.41 and $1716.53 \mathrm{~cm}^{-1}$, and concomitant shift to higher frequencies of urea carbonyl stretching vibration at 1618.2 and 1521.73 to 1635.5 and $1558.38 \mathrm{~cm}^{-1}$, respectively.
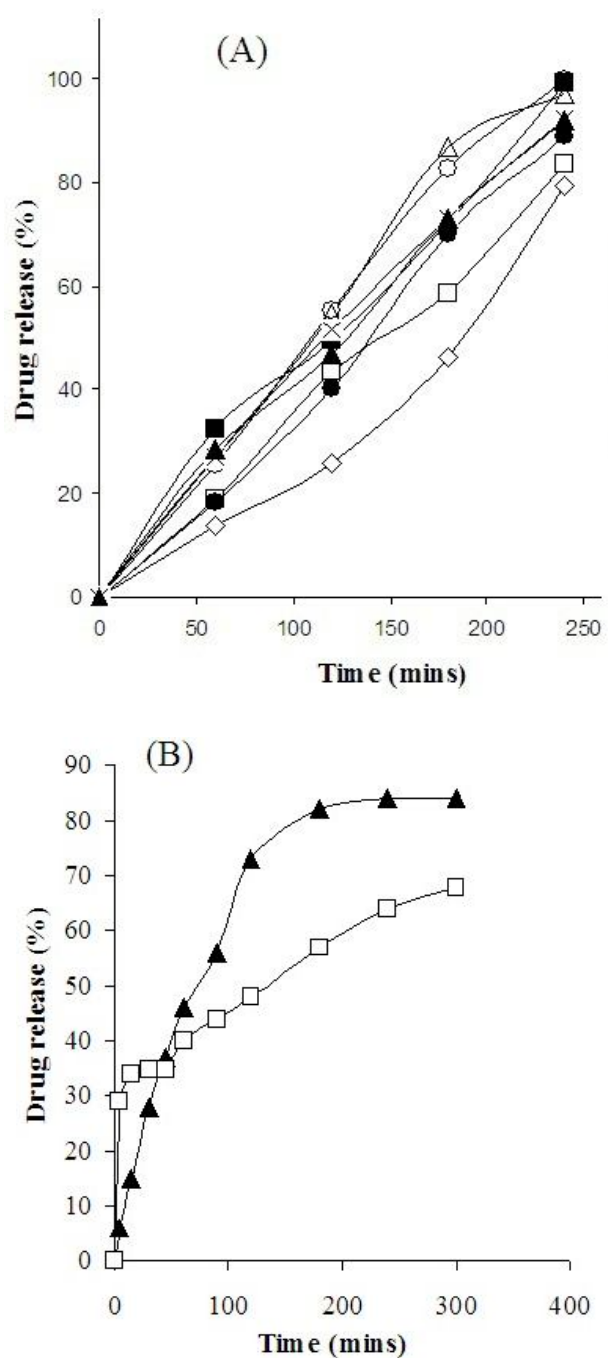

Fig 3: Comparative in vitro drug release of $(A) S D$ formulations $(\diamond=\mathrm{G} 1, \square=\mathrm{G} 2, \circ=\mathrm{G} 3, \Delta=\mathrm{G} 4 . \square=$ $\mathrm{G} 5, \bullet=\mathrm{G} 6, \times=\mathrm{G} 7, \boldsymbol{\Delta}=\mathrm{G} 8$; and (B) optimized $\mathrm{SD}(\boldsymbol{\Lambda})$ and a commercial GLB brand, Betanase $\AA$ (口). 
Upadhyay \& Pandit

Table 2: Release kinetic data for GLB solid dispersions (SDs) based on various models

\begin{tabular}{cccccc}
\hline \multirow{2}{*}{$\begin{array}{c}\text { Formulation } \\
\text { code }\end{array}$} & $\begin{array}{c}\text { First } \\
\text { order }\end{array}$ & $\begin{array}{c}\text { Higuchi } \\
\text { model }\end{array}$ & $\begin{array}{c}\text { Zero } \\
\text { order }\end{array}$ & \multicolumn{2}{c}{ Korsmeyer-Peppas } \\
\hline GI & 0.6893 & 0.9531 & 0.9874 & 0.9116 & $\mathbf{r}$ \\
G II & 0.6673 & 0.9152 & 0.9707 & 0.8939 & 0.8378 \\
G III & 0.7200 & 0.9463 & 0.9722 & 0.9266 & 0.8398 \\
G IV & 0.8005 & 0.9265 & 0.9888 & 0.9714 & 0.8384 \\
G V & 0.7238 & 0.9750 & 0.9611 & 0.9334 & 0.8246 \\
G VI & 0.7506 & 0.9210 & 0.9710 & 0.9462 & 0.9221 \\
G VII & 0.6927 & 0.9732 & 0.9742 & 0.9131 & 0.9180 \\
G VIII & 0.6959 & 0.9585 & 0.9915 & 0.9162 & 0.8258 \\
G IX & 0.7072 & 0.8990 & 0.9288 & 0.9135 & 0.8119 \\
\hline
\end{tabular}

Note: Formulation code as in Table $1 ; r^{2}=$ regression coefficient.

\section{In vitro buoyancy, and drug entrapment and release}

In vitro release profiles of the GLB SDs formulation in simulated gastric $(\mathrm{pH} \mathrm{1.2)}$ are shown in Fig 3A). In vitro buoyancy results indicate that the SD formulation remained floating for $11 \mathrm{~h}$ while drug entrapment efficiency was as high as $99.8 \%$. The regression coefficient $\left(r^{2}\right)$ data based on kinetic analysis using various release models are listed in Table 2. The formulations were best fitted to the zero-order release model, with $r^{2}$ close to one. Korsmeyer-Peppas $n$ data also indicate that the drug release mechanism was non-Fickian case II diffusioncontrolled with $n$ values ranging from 0.80 to 0.9221). The in vitro release data of optimized test GLB solid dispersion and commercial reference brand (Betanase ${ }^{\circledR}$ tablet) are indicated in Fig 3B. Release profiles of the two preparations were comparable.

\section{Effect of ageing}

When the GLB SDs were kept at $30^{\circ} \mathrm{C} / 65$ $\% \mathrm{RH}$ for 3 months, no change in in vitro drug dissolution was observed, compared with the initial release rate.

\section{Effect of GLB SDs on blood glucose level}

As shown in Fig 4 the hypoglycemia produced in diabetic treated group IV treated with SD was significantly higher $(p<0.01)$ than the diabetic standard (GLB) treated group III. The Blood glucose level in diabetic treated group IV in $4 \mathrm{~h}$ were the same with Control group I compared with diabetic group II and diabetic standard treated group III.

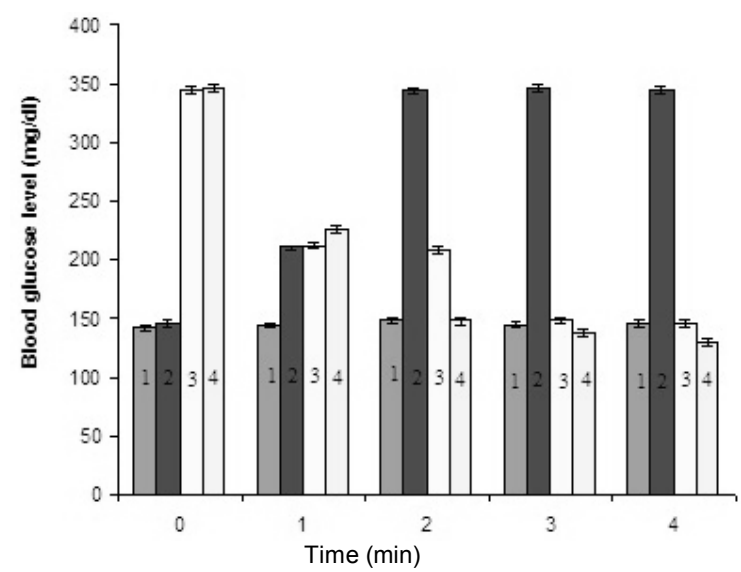

Fig 4: In vivo blood glucose lowering properties of optimized SD formulation in wistar rats. Key: (1) = Normal control, $(2)=$ Diabetic control II, (3) = Diabetic standard treatment, and (4) Diabetic SD treatment 


\section{DISCUSSION}

Hot melt granulation technique was selected to achieve solid dispersion, as it has been successfully utilized to increase the solubility of GLB [1]. PEGs and Gelucire are among the several carriers that have been employed in preparing solid dispersions.

The purpose of the current study was to examine the solid-state properties of a solid dispersion system of GLB prepared using Gelucire 50/13 and various grades of PEGs at varying ratios. Intermolecular interaction such as hydrogen bonding between the amide of GLB and the oxygen of polyglycol chain (Gelucire) inducing a shift of $\mathrm{N}-\mathrm{H}$ vibration to an extent that depends on the strength of interaction. The site of the interaction on Gelucire would probably have been $\mathrm{C}=\mathrm{O}$ group, which would also affect $\mathrm{N}$ $\mathrm{H}$ vibration. This observation agrees with the data generated from PXRD and FTIR studies.

Both HSPM and x-ray diffraction analysis revealed that GLB was in an amorphous state and uniformly distributed throughout matrix while FTIR spectroscopy revealed the possibility of $\mathrm{H}$-bonding interaction in both PMs and SDs.

To achieve gastro-retention, the time needed for the initiation of floatation (floating lag time) was less than $3 \mathrm{~min}$ for all the SD formulations. However, maximum in vitro buoyancy was $11 \mathrm{~h}$. SDs exhibited dramatically improvement in initial rate as well as extent of in vitro drug dissolution. Zero-order kinetics model fitted best for the formulations while the $n$ exponent of the Korsmeyer-Peppas model indicate that erosion was the mechanism of drug release. Release of drug from hydrophilic minimatrices generally involves both pore diffusion and matrix erosion [5]. Low density and hydrophilic property due to the higher HLB of Gelucire 50/13 facilitated gastroretension and drug dissolution. Gelucire 50/13 possesses surfactant and self-emulsifying property and is also used as meltable binder by melt granulation of poorly water soluble active substances [9]. In contact with aqueous fluids, it forms a fine emulsion, solubilizes the active substance and hence increases its oral bioavailability.

Incorporation of GLB in the Gelucire 50/13 led to the formation of a solid dispersion system with increased dissolution rate of the drug due to improved wettability and the additional presence of a self-emulsifying compound, PEG. Moreover, it also increased the dispersability of the hydrophobic drug in the hydrophilic carrier during the process of solid dispersion formation. Thus, the increase in the dissolution of the drug can be attributable to improvement in wetting and to local solubilization by the excipients in the diffusion layer. The SDs exhibited higher burst release due probably to enhanced wettability of the drug particles, the emulsifying effect of carriers, significant reduction in particle size during SD formation and/or the inherently higher dissolution rate of soluble component of the SDs, which would pull along the more insoluble but finely mixed drug into the dissolution medium. Improved drug dissolution could also be attributed to the presence of the amorphous form of GLB, as indicated by the x-ray diffraction findings.

The increase in the release rate of the GLB SDs was also reflected in vivo by the greater reduction in blood glucose level in Wistar rats by GLB SDs, compared to pure GLB.

\section{CONCLUSION}

The present study demonstrates the high potential of hot melt-granulation technique for the production of solid dispersions of glibenclamide using polyglycolized glycerides as carriers. However, further studies are required to develop the formulation to industrial scale production.

\section{COMPETING INTEREST}

The authors declare no conflict of interest. 


\section{ACKNOWLEDGEMENT}

One of the authors sincerely thanks to Dr. RM Dubey, Director, I.F.T.M for providing all the research facilities. The authors are also thankful to Gattefosse, France for providing Gelucires free of charge and to Instrumental lab, IIT-Roorkee for x-ray diffraction and DSC studies. This paper is based, in part, on the work done for a PhD degree of G.B. Technical University, Lucknow, India by one of the authors (Prashant Upadhyay).

\section{REFERENCES}

1. Fukuda M, Peppas NA, Mcginity JW, Floating hot melt extruded tablets for gastroretentive controlled drug release system, J. Control. Release. 2006; 115: 121-129.

2. Lee JW, Park JH, Robinson JP, Bioadhesive based dosage forms, the next generation, J. Pharm. Sci. 2000; 89: 850-866.

3. Bardonnet PL, Faiere V, Jpugh W, Gastroretentive dosage forms: overview and special case of helicobacter pylori, J. Control. Release. 2006; 111: 1-18.

4. Strewbel A, Siepman J, Bodmeier R, Gastroretentive drug delivery system, Expert Opin. Drug Deliv. 2006; 3: 217-233.

5. Hauss DJ, Oral lipid-based formulations, Adv. Drug Deliv. Rev. 2007; 59: 667-676.

6. Jannin V, Musakhanian J, Marchaud D, Approaches for the development of solid and semi-solid lipid-based formulations, Adv. Drug Deliv. Rev. 2008; 60: 734-746.

7. Chakraborty S, Shukla D, Mishra B, Singh S, Lipid An emerging platform for oral delivery of drugs with poor bioavailability, Eur. J. Pharm. Biopharm. 2009; 73: 1-15.

8. Kumar K, Shah MH, Ketkar AR, Kadam SS, Paradkar $A$, Effect of drug solubility and different excipients on floating behaviour and release from glyceryl monooleate matrices, Int. J. Pharm. 2004; 272: 151-160.

9. Finia A, Moyanob JR, Gine'sb JM, Perez-Martinezb Jl, Rabasco AM, Diclofenac salts II Solid dispersions in PEG6000 and Gelucire 50/13, Eur. J. Pharm. Biopharm 2005; 60: 99-111.

10. Kawakami K, Oda N, Miyoshi K, Funaki T, Ida Y, Solubilization behavior of a poorly soluble drug under combined use of surfactants and cosolvents, Eur. J. Pharm. Sci. 2008; 28: 714.

11. Porter CJH, Pouton CW, Cuine JF, Charman WN, Enhancing intestinal drug solubilisation using lipid-based delivery systems, Adv. Drug Deliv. Rev. 2008; 60: 673-691.

12. Urbanetz NA, Lippold BC, Solid dispersions of nimodipine and polyethylene glycol 2000: dissolution properties and physico-chemical characterization, Eur. J. Pharm. Biopharm. 2005; 59: 107-118.

13. Unger J, Tajarobi F, Norder O, Frenning G, Larsson $A$, Relating solubility data of parabens in liquid PEG 400 to the behaviour of PEG 4000parabens solid dispersions, Eur. J. Pharm. Biopharm. 2009; 73: 260-268.

14. Varma MM, Pandit JK, Dissolution, Solubility, XRD and DSC studies on Flurbiprofen-Nicotinamide Solid Dispersions, Drug Development and Industrial Pharmacy 2005; 31: 417-423.

15. Higuchi $T$, Mechanism of sustained release medication: theoretical analysis of rate of release of solid drugs dispersed in solid matrices, J. Pharm. Sci. 1963; 52: 1145-1149.

16. Korsmeyer RW, Gunny R, Peppas NA, Mechanism of solute release from hydrophilic polymers, Int. J. Pharm. 1983; 15: 25-35.

17. Srinatha $A$, Pandit JK, Singh S, lonic cross-linked chitosan beads for extended release of ciprofloxacin: In vitro characterization, Indian J. Pharm. Sci. 2008; 70: 16-21.

18. Ministry of Environment and Forests, Government of India. Public information: Good Practices: CPCSEA guidelines; [updated 2004 May 12; cited 2009 May 14]. Available from: http://moef.nic.in 